\title{
Local Stability of a New Adaptive Queue Management (AQM) Scheme
}

\author{
Li Zhu, Student Member, IEEE, and Nirwan Ansari, Senior Member, IEEE
}

\begin{abstract}
In this letter, we propose a new Adaptive Queue Management scheme based on the virtual queue size and the aggregate flow rate. Our proposal is tailored for the widely deployed TCP Reno and does not require modifications to TCP end users. We prove that our scheme can guarantee local stability for a network with an arbitrary topology and heterogeneous round-trip times for TCP users.
\end{abstract}

Index Terms-Adaptive Queue Management (AQM), stability, TCP, virtual queue.

\section{INTRODUCTION}

A CTIVE Queue Management (AQM) has been a very active research area in recent years. Many AQM mechanisms have been proposed, e.g., random early detection (RED) [1], random exponential marking (REM) [2], PI controller [3], adaptive virtual queue (AVQ) [4], and state feedback control (SFC) [5]. AQM schemes control the traffic rate and buffer occupancy by schematically dropping packets. If Explicit Congestion Control (ECN) is enabled, packets are marked instead of being dropped. Without loss of generality, we assume ECN is enabled in this letter. End TCP users adapt their transmission rates based on the marking feedback from AQM.

RED is a queue length based AQM that marks packets with probability proportional to the current average queue length. AVQ is a typical rate based scheme. REM, PI, and SFC use the queue length and the aggregate flow rate to compute the marking probability.

The TCP/AQM system has been modeled as a close loop control system. One of the major concerns about such a system is its stability property. [3]-[5] studied the local stability conditions for the network with PI, AVQ, and SFC, respectively. These works only considered a network of a single bottleneck link with homogeneous round-trip times, and neglected the backward propagation delays. [6] took the heterogeneous round-trip time and backward propagation delay into consideration, and provided the local stability condition for RED in a network with a single bottleneck link.

In this letter, we propose a virtual queue and rate based AQM scheme (referred to as VQR), which uses the virtual queue size

\footnotetext{
Manuscript received November 3, 2003. The associate editor cordinating the review of this letter and approving it for publication was Prof. D. Petr. This work has been supported in part by the New Jersey Commission on Higher Education under the NJI-TOWER project, and by the New Jersey Commission on Science and Technology under NJWINS.

The authors are with the Advanced Networking Laboratory, Department of Electrical and Computer Engineering, New Jersey Institute of Technology, Newark, NJ07102 USA (e-mail: 1z6@njit.edu; nirwan.ansari@njit.edu). Digital Object Identifier 10.1109/LCOMM.2004.831335
}

and the aggregate flow rate to compute the marking probability. We provide and prove the local stability condition of our scheme for a network with an arbitrary topology rather than a single bottleneck link, with heterogeneous rather than homogeneous round-trip times, and with the consideration of rather than negligence of the backward propagation delays.

The remainder of this letter is organized as follows. Section II presents the framework of the TCP/AQM dynamic model. Section III presents our proposed VQR scheme and the corresponding local stability condition. Section IV presents the conclusions.

\section{TCP/AQM DYNAMICS}

In this section, we adopt the work in [6] to present the TCP/AQM dynamic model used in this letter. Consider a network with $L$ links, each with capacity $C_{l}(l=1,2, \ldots L)$. Assume there exist $S$ TCP sources, which share the $L$ links. The routing policy is expressed by an $L \times S$ matrix $\mathbf{R}$ with elements $R_{l i}$ defined as

$$
R_{l i}= \begin{cases}1, & \text { if source } i \text { uses link } l \\ 0, & \text { otherwise. }\end{cases}
$$

Each link $l$ marks packets with probability $p_{l}(t)$ at time $t$. Each TCP source $i$ is associated with a round-trip time $\tau_{i}(t)$

$$
\tau_{i}(t)=d_{i}+\sum_{l} R_{l i} \frac{b_{l}(t)}{C_{l}}
$$

where $d_{i}$ is the round-trip propagation delay for source $i$ and $b_{l}(t)$ is the queue length of link $l$ at time $t$. The round-trip time delay $\tau_{i}(t)$ can also be expressed as

$$
\tau_{i}(t)=\tau_{l i}^{f}(t)+\tau_{l i}^{b}(t)
$$

where $\tau_{l i}^{f}(t)$ is the forward delay from source $i$ to link $l$ and $\tau_{l i}^{b}(t)$ is the backward delay from link $l$ back to source $i$.

The transmitting rate of source $i, x_{i}(t)$, is modeled as

$$
x_{i}(t)=\frac{w_{i}(t)}{\tau_{i}(t)}
$$

where $w_{i}(t)$ is the window size of $i$. The aggregate transmission rate at link $l$ is

$$
y_{l}(t)=\sum_{i} R_{l i} x_{i}\left(t-\tau_{l i}^{f}(t)\right) .
$$

With the assumption that $p_{l}(t)$ is small, the end-to-end marking probability $q_{i}(t)$ for source $i$ can be obtained by summing up the marking probability at each link traversed by $i$

$$
q_{i}(t)=\sum_{l} R_{l i} p_{l}\left(t-\tau_{l i}^{b}(t)\right) .
$$


We adopt the fluid TCP model studied in [3] and [6], in which the TCP Reno window size evolves according to

$$
\dot{w}_{i}(t)=\frac{x_{i}\left(t-\tau_{i}(t)\right)\left(1-q_{i}(t)\right)}{w_{i}(t)}-x_{i}\left(t-\tau_{i}(t)\right) q_{i}(t) \frac{w_{i}(t)}{2} .
$$

The first term on the right side of (7) describes the additive window increment, and the second term stands for the multiplicative window decrement. The sending rate of TCP $i$ evolves according to

$$
\dot{x}_{i}(t) \triangleq \frac{d}{d t}\left(\frac{w_{i}(t)}{\tau_{i}(t)}\right)=\frac{\dot{w}_{i}(t)}{\tau_{i}(t)}-\frac{w_{i}(t) \dot{\tau}_{i}(t)}{\tau_{i}^{2}(t)} .
$$

The dynamics of the queue length $b_{l}(t)$ at link $l$ can be expressed as

$$
\dot{b}_{l}(t)=y_{l}(t)-C_{l} \text {. }
$$

Denote $w_{i}^{*}, p_{l}^{*}, q_{i}^{*}$ as the equilibrium values of $w_{i}(t), p_{l}(t)$, $q_{i}(t)$, respectively. At equilibrium, we have $x_{i}^{*}=w_{i}^{*} \tau_{i}$, and $q_{i}^{*}=\sum_{l} R_{l i} p_{l}^{*}$. Here, $\tau_{i}=d_{i}+\sum_{l} R_{l i}\left(b_{l}^{*} / C_{l}\right)$ is the equilibrium round-trip time for source $i$. It can be shown from (7) that

$$
w_{i}^{*}=\sqrt{\frac{2\left(1-q_{i}^{*}\right)}{q_{i}^{*}}} .
$$

Let $\delta w_{i}(t)=w_{i}(t)-w_{i}^{*}, \delta p_{l}(t)=p_{l}(t)-p_{l}^{*}, \delta x_{i}(t)=$ $x_{i}(t)-x_{i}^{*}$. Linearizing (7), (8), and (5) around the equilibrium state with the first-order approximation, we have

$$
\begin{aligned}
\delta \dot{w}_{i}(t) & =-\frac{1}{\tau_{i} q_{i}^{*}} \sum_{l} R_{l i} \delta p_{l}\left(t-\tau_{l i}^{b}\right)-\frac{q_{i}^{*} w_{i}^{*}}{\tau_{i}} \delta w_{i}(t) \\
\delta \dot{x}_{i}(t) & =\frac{\delta \dot{w}_{i}(t)}{\tau_{i}}-\frac{w_{i}^{*} \delta \dot{\tau}_{i}}{\tau_{i}^{2}} \\
\delta \dot{y}_{l}(t) & =\sum_{i} R_{l i} \delta \dot{x}_{i}\left(t-\tau_{l i}^{f}\right) .
\end{aligned}
$$

\section{THE VQR ALGORITHM}

VQR maintains a virtual queue $B_{l}(t)$, which is updated at each packet arrival according to

$$
\dot{B}_{l}(t)=y_{l}(t)-\gamma C_{l}
$$

where $\gamma$ is a positive number less than but close to 1 (e.g., 0.95). The marking probability is updated by

$$
p_{l}(t)=\frac{g}{\gamma C_{l}}\left\{a\left(B_{l}(t)-B_{l, r e f}\right)+\left(y_{l}(t)-\gamma C_{l}\right)\right\}
$$

where $B_{l, r e f}$ is a reference virtual queue length, and $g$ and $a$ are positive numbers. If $p_{l}(t)<0$, it is set to zero; if $p_{l}(t)>1$, it is set to one.

At the equilibrium state, we have $\dot{B}_{l}(t)=0$. Thus, the aggregate rate $y_{l}^{*}$ is $\gamma C_{l}$, which is slightly smaller than the link capacity $C_{l}$. Although this could lead to slight link under-utilization, there are some benefits of doing so. First, the queuing delay is zero at the equilibrium state. Second, since we only consider small perturbation around the equilibrium state, it can reasonably be assumed that $y_{l}(t)$ is always less than the link capacity. As a result, the queue length $b_{l}(t)$ is always zero, the round-trip time $\tau_{i}(t)$ is reduced to $d_{i}$, and $\delta \tau_{i}(t)$ is always zero under such an assumption. Thus, the second term on the right side of (12) is negligible. Together with (11), we can rewrite (12) as

$$
\begin{aligned}
\delta \dot{x}_{i}(t) & =\frac{\delta \dot{w}_{i}(t)}{\tau_{i}} \\
& =-\frac{1}{\tau_{i}^{2} q_{i}^{*}} \sum_{l} R_{l i} \delta p_{l}\left(t-\tau_{l i}^{b}\right)-\frac{q_{i}^{*} w_{i}^{*}}{\tau_{i}} \delta x_{i}(t) .
\end{aligned}
$$

Linearizing (14) and (15) around the equilibrium state, we have

$$
\begin{aligned}
\delta \dot{B}_{l}(t) & =\delta y_{l}(t), \\
\delta p_{l}(t) & =\frac{g}{\gamma C_{l}}\left(a \delta B_{l}(t)+\delta y_{l}(t)\right) .
\end{aligned}
$$

Following [6], we can express (13), and (16)-(18) in the Laplace domain in the matrix form:

$$
\begin{aligned}
& \boldsymbol{\delta} \mathbf{y}(s)=\mathbf{R}_{\mathbf{f}}(s) \boldsymbol{\delta} \mathbf{x}(s), \\
& \boldsymbol{\delta} \mathbf{x}(s)=-\left(s \mathbf{I}+\mathbf{H}_{\mathbf{1}}\right)^{-1} \mathbf{H}_{\mathbf{2}} \mathbf{R}_{\mathbf{b}}^{\mathbf{T}}(s) \boldsymbol{\delta} \mathbf{p}(s), \\
& \boldsymbol{\delta} \mathbf{p}(s)=\mathbf{A}(s) \mathbf{G} \boldsymbol{\delta} \mathbf{y}(s)
\end{aligned}
$$

where $\mathbf{G}=\operatorname{diag}\left(g / \gamma C_{l}\right), \mathbf{A}(s)=\operatorname{diag}(s+a / s), \mathbf{H}_{\mathbf{1}}=$ $\operatorname{diag}\left(q_{i}^{*} w_{i}^{*} / \tau_{i}\right), \mathbf{H}_{2}=\operatorname{diag}\left(1 / \tau_{i}^{2} q_{i}^{*}\right)$, and

$$
\begin{aligned}
{\left[R_{f}\right]_{l i} } & = \begin{cases}e^{-\tau_{l i}^{f} s}, & \text { if source } i \text { uses link } l \\
0, & \text { otherwise }\end{cases} \\
{\left[R_{b}\right]_{l i} } & = \begin{cases}e^{-\tau_{l i}^{b} s}, & \text { if source } i \text { uses link } l \\
0, & \text { otherwise. }\end{cases}
\end{aligned}
$$

Equations (19)-(21) form a closed-loop control system with the return ratio

$$
\mathbf{L}(s)=\mathbf{R}_{\mathbf{f}}(s)\left(s \mathbf{I}+\mathbf{H}_{\mathbf{1}}\right)^{-1} \mathbf{H}_{\mathbf{2}} \mathbf{R}_{\mathbf{b}}^{\mathbf{T}}(s) \mathbf{A}(s) \mathbf{G} .
$$

According to [6] and [7], the above control system is stable if the eigenvalues of $L(j \omega)(\omega \geq 0)$ do not encircle -1 in the complex plane. Define

$$
\tilde{\mathbf{R}}(j \omega)=\operatorname{diag}\left(\sqrt{\frac{g}{\gamma C_{l}}}\right) \mathbf{R}_{\mathbf{f}}(j \omega) \operatorname{diag}\left(\sqrt{x_{i}^{*}}\right) .
$$

Using the relationship

$$
\mathbf{R}_{\mathbf{b}}(s)=\mathbf{R}_{\mathbf{f}}(-s) \operatorname{diag}\left(e^{-\tau_{i} s}\right)
$$

and following the similar argument in [7], the eigenvalues of $L(j \omega)$ are the same as those of:

$\mathbf{Z}(j \omega)=\operatorname{diag}\left(\frac{e^{-j \omega \tau_{i}}(j \omega+a)}{q_{i}^{*} x_{i}^{*}\left(j \omega \tau_{i}+q_{i}^{*} w_{i}^{*}\right) j \omega \tau_{i}}\right) \tilde{\mathbf{R}}^{\mathbf{T}}(-j \omega) \tilde{\mathbf{R}}(j \omega)$.

Denote $E$ as the set of eigenvalues of $Z(j \omega)$. According to [7], we have

$$
\begin{aligned}
& E \subset \rho\left(\tilde{\mathbf{R}}^{\mathbf{T}}(-j \omega) \tilde{\mathbf{R}}(j \omega)\right) \\
& \times c o\left(0 \cup\left(\frac{e^{-j \omega \tau_{i}}(j \omega+a)}{q_{i}^{*} x_{i}^{*}\left(j \omega \tau_{i}+q_{i}^{*} w_{i}^{*}\right) j \omega \tau_{i}}\right),\right. \\
& \quad i=1,2, \ldots, S)
\end{aligned}
$$


where $c o\left(m_{i}, i=1,2, \ldots, S\right)$ denotes the convex hull of the set points $\left\{m_{1}, m_{2}, \ldots, m_{S}\right\}$. The spectral radius of $\tilde{R}(j \omega)$ satisfies [7]:

$$
\begin{aligned}
& \rho\left(\tilde{\mathbf{R}}^{\mathbf{T}}(-j \omega) \tilde{\mathbf{R}}(j \omega)\right) \\
& \quad \leq\left\|\mathbf{R}_{\mathbf{f}}^{\mathbf{T}}(-j \omega) \operatorname{diag}\left(\frac{g}{\gamma C_{l}}\right) \mathbf{R}_{\mathbf{f}}(j \omega) \operatorname{diag}\left(x_{i}^{*}\right)\right\|_{\infty} \\
& \quad \leq\left\|\mathbf{R}_{\mathbf{f}}^{\mathbf{T}}(-j \omega)\right\|_{\infty} \cdot\left\|\operatorname{diag}\left(\frac{g}{\gamma C_{l}}\right) \mathbf{R}_{\mathbf{f}}(j \omega) \operatorname{diag}\left(x_{i}^{*}\right)\right\|_{\infty} \\
& \quad=M g
\end{aligned}
$$

where $M$ is the maximum number of links a TCP source traverses in the network.

Denote

$$
\Lambda_{i}(j \omega)=\frac{M g \times e^{-j \omega \tau_{i}}(j \omega+a)}{q_{i}^{*} x_{i}^{*}\left(j \omega \tau_{i}+q_{i}^{*} w_{i}^{*}\right) j \omega \tau_{i}} .
$$

If $\Lambda_{i}(j \omega)(i=1,2, \ldots, M)$ do not encircle -1 in the complex plane, $E$ does not either [7]. Therefore, the system is stable. Next, we present a sufficient condition to guarantee the stability of such a system.

Theorem 1: If

$$
a<\min _{i}\left\{\left(1 / \tau_{i}\right) \sqrt{2 q_{i}^{*}\left(1-q_{i}^{*}\right)} ; i=1, \ldots, S\right\}
$$

and $g<(\pi / 2 M) \min _{i}\left(\sqrt{2 q_{i}^{*}\left(1-q_{i}^{*}\right)} ; i=1, \ldots, S\right)$, the system is stable.

Proof: From (30), we can rewrite $\Lambda_{i}(j \omega)$ as

$$
\Lambda_{i}(j \omega)=\frac{e^{-j \omega \tau_{i}}\left(j \omega \tau_{i}+a \tau_{i}\right)}{j \omega \tau_{i}\left(j \omega \tau_{i}+q_{i}^{*} w_{i}^{*}\right)} \frac{M g}{q_{i}^{*} w_{i}^{*}} .
$$

With (10), it can be shown that the two conditions in Theorem 1 are equivalent to

$$
\begin{aligned}
& a<\min _{i}\left\{\frac{1}{\tau_{i}} q_{i}^{*} w_{i}^{*}, i=1, \ldots, S\right\} \\
& g<\frac{\pi}{2 M} \min _{i}\left(q_{i}^{*} w_{i}^{*}, i=1, \ldots, S\right) .
\end{aligned}
$$

Let

$$
\frac{e^{-j \omega \tau_{i}}}{j \omega \tau_{i}} \frac{\left(j \omega \tau_{i}+a \tau_{i}\right)}{\left(j \omega \tau_{i}+q_{i}^{*} w_{i}^{*}\right)}=\rho e^{j \theta} \frac{e^{-j \omega \tau_{i}}}{j \omega \tau_{i}}
$$

where

$$
\begin{aligned}
& \theta=\operatorname{phase}\left(\left(j \omega \tau_{i}+a \tau_{i}\right) /\left(j \omega \tau_{i}+q_{i}^{*} w_{i}^{*}\right)\right) \\
& \rho=\left|\left(\left(j \omega \tau_{i}+a \tau_{i}\right) /\left(j \omega \tau_{i}+q_{i}^{*} w_{i}^{*}\right)\right)\right| .
\end{aligned}
$$

If $a<\min _{i}\left\{\left(q_{i}^{*} w_{i}^{*} / \tau_{i}\right) ; i=1, \ldots, S\right\}$, we have $0<\theta<$ $\pi / 2$ and $0<\rho<1$. Let $\omega^{\prime}$ be any frequencies at which $\rho e^{j \theta}\left(e^{-j \omega \tau_{i}} / j \omega \tau_{i}\right)$ cross the negative real axis, and we have

$$
-\omega^{\prime} \tau_{i}-\frac{\pi}{2}+\theta=-(2 k+1) \pi, \quad k=0,1,2, \ldots
$$

Thus, from (34) and (35) we have

$$
\frac{e^{-j \omega^{\prime} \tau_{i}}}{j \omega^{\prime} \tau_{i}} \frac{\left(j \omega^{\prime} \tau_{i}+a \tau_{i}\right)}{\left(j \omega^{\prime} \tau_{i}+q_{i}^{*} w_{i}^{*}\right)}=-\frac{\rho}{\left(2 k+\frac{1}{2}\right) \pi+\theta} .
$$

From (35) and with the properties of $\rho$ and $\theta$, we have

$$
\frac{e^{-j \omega^{\prime} \tau_{i}}}{j \omega^{\prime} \tau_{i}} \frac{\left(j \omega^{\prime} \tau_{i}+a \tau_{i}\right)}{\left(j \omega^{\prime} \tau_{i}+q_{i}^{*} w_{i}^{*}\right)}>-\frac{2}{\pi} .
$$

If $g<(\pi / 2 M) \min _{i}\left(q_{i}^{*} w_{i}^{*} ; i=1, \ldots, S\right)$, from (36) we have

$$
\frac{e^{-j \omega^{\prime} \tau_{i}}}{j \omega^{\prime} \tau_{i}} \frac{\left(j \omega^{\prime} \tau_{i}+a \tau_{i}\right)}{\left(j \omega^{\prime} \tau_{i}+q_{i}^{*} w_{i}^{*}\right)} \frac{M g}{q_{i}^{*} w_{i}^{*}}>-1 .
$$

In other words, $\Lambda_{i}(j \omega)$ only cross the real axis on the right side of point -1 , and thus never encircle -1 in the complex plane. Therefore, the system is stable.

\section{CONCLUSIONS}

In this letter, we have proposed VQR, a new AQM scheme based on the virtual queue length and the aggregate flow rate. VQR can achieve high link utilization and near zero queuing delay. We have also presented and proved the local stability condition for a TCP/VQR based network with an arbitrary topology, heterogeneous round-trip times, and backward propagation delays.

\section{REFERENCES}

[1] S. Floyd and V. Jacobson, "Random early detection gateways for congestion avoidance," IEEE/ACM Trans. Networking, vol. 1, no. 4, pp. 397-413, Aug. 1993.

[2] S. Athuraliya, S. H. Low, V. H. Li, and Q. Yin, "REM: Active queue management," IEEE Network Magazine, vol. 15, no. 3, pp. 48-53, May/June 2001.

[3] C. Hollot, V. Misra, D. Towsley, and W. Gong, "On designing improved controllers for AQM routers supporting TCP flows," in Proc. IEEE INFOCOM 2001, vol. 3, AK, Apr. 2001, pp. 1726-1734.

[4] S. Kunniyur and R. Srikant, "Analysis and design of an active virtual queue (AVQ) algorithm for active queue management," in Proc. ACM SIGCOMM 2001, San Diego, CA, Aug. 2001, pp. 123-134.

[5] Y. Gao and J. C. Hou, "A state feedback control approach to stabilizing queues for ECN-enabled TCP connections," in Proc. IEEE INFOCOM 2003, vol. 3, San Diego, CA, Apr. 2003, pp. 2301-2311.

[6] S. H. Low, F. Paganini, J. Wang, and J. C. Doyle, "Linear stability of TCP/RED and a scalable control," Computer Networks J., vol. 43, no. 5, pp. 633-647, Dec. 2003.

[7] G. Vinnicombe, "On the scalability of end-to-end congestion control for the Internet," Cambridge Univ., Cambridge, U.K., CUED/F-INFENG/TR.398, 2000. 